Revista Calidad en la Educación Superior

Programa de Autoevaluación Académica

Universidad Estatal a Distancia, Costa Rica

ISSN 1659-4703

revistacalidad@uned.ac.cr

\title{
LA EDUCACIÓN EN EL CONTEXTO DE LA SOCIEDAD ACTUAL
}

\author{
EDUCATION IN THE CONTEXT OF MODERN SOCIETY
}

\author{
Ingrid L. Ambrosy Velarde ${ }^{1}$ \\ iambrosy@url.edu.gt \\ Universidad Rafael Landívar de Guatemala
}

\author{
Volumen 6, Número 2 \\ Noviembre 2015 \\ Pp. $200-213$
}

Recibido: 25 de enero, 2014

Aprobado: 30 de setiembre, 2015

\footnotetext{
${ }^{1}$ Licenciada en Psicología, con una Maestría en Docencia Universitaria. Candidata a Doctora en Educación, por UNED, promoción XIII. Se desempeña como Directora de la Dirección de Desarrollo y Actualización Académicos en la Universidad Rafael Landívar de Guatemala. Correo electrónico: iambrosy@url.edu.gt
} 


\title{
Resumen
}

El contexto de la sociedad actual se desarrolla en medio de una corriente de avances tecnológicos, científicos y productivos, y otra de aplazamiento de la justicia, la igualdad y la paz. En algunas regiones, como Latinoamérica, aún hace falta mucho por hacer para reducir problemas que tanto afectan la calidad de vida de sus habitantes y lo alejan de una convivencia pacífica.

Ante estas situaciones, la sociedad necesita tomar consciencia de qué tipo de ciudadano espera formar y además, qué tipo de competencias tiene que promover a través del sistema educativo. Para lograrlo, es preciso que se unan estado, sector productivo, gestores académicos, profesores y comunidad, para contar con una base económica, política y administrativa, así como con las herramientas para formar competencias elementales tales como el conocimiento personal y social, la autoevaluación, renovación, solidaridad, identidad local y mundial, el aprender a aprender, la convivencia, enfrentar la complejidad, conciencia del deterioro ambiental y habilidades para la producción científica, que se reflejarán en ciudadanos comprometidos con la paz y la igualdad de la región.

Palabras clave: fines de la educación, educación para la paz.

\begin{abstract}
The context of contemporary society unfolds amid a stream of technological, scientific and production advances and other deferral of justice, equity and peace. In some regions, such as Latin America is still needed to be done reducing both problems that affects the quality of life of its inhabitants that draw them away from peaceful coexistence.

In these situations, society needs to become aware of what kind of city hopes to form and also what kind of skills you need to advance through the educational system. To achieve this, we need to join state, productive sector, academic managers, teachers and community, to be counted with a political and administrative economic base, as well as tools to form basic skills such as personal and social knowledge self-assessment, renovation, solidarity, global and local identity, learning to learn, coexist, facing to complexity, awareness of environmental degradation and skills to scientific production, which will be reflected in committed to equity and peace citizens in the region .
\end{abstract}

Keywords: Purposes of Education; Education for peace. 
A través de este artículo, se hace una reflexión sobre aquellos aprendizajes que deben promoverse a través de la educación, en medio de una época en la que el desarrollo humano en Latinoamérica, y especialmente en Centroamérica, sigue quedando rezagado en comparación con otros países en desarrollo o en vías de desarrollo a nivel mundial.

Se inicia con una revisión de los ámbitos en los que se desenvuelve ser humano de hoy, la realidad educativa de los países latinoamericanos, para luego analizar las competencias que necesita para sobrevivir y para hacer las transformaciones que coadyuven a que el mundo sea un lugar más habitable para él y las futuras generaciones. Finalmente, se invita a quienes se desempeñan en cualquier nivel educativo a repensar en los fines de la educación y a tomar las acciones necesarias para que la gestión educativa, el currículo, las metodologías, la evaluación, el rol del profesor y del estudiante sean coherentes con las intenciones de formar ciudadanos para ese mundo que se anhela, de paz y desarrollo sustentable.

La sociedad del siglo XXI está caracterizada por cambios en las diferentes áreas de desarrollo, como lo son la económica, cultural, política, social, ambiental y biológica, entre otras. De acuerdo con Castells (2004), en la última parte del milenio pasado hubo transformaciones sociales multidimensionales, esto quiere decir en distintos ámbitos del desarrollo y actuación, tales como: factores demográfico, la expectativa de vida, las relaciones de poder, la estructura familiar, el ritmo de producción y empleabilidad, el desarrollo tecnológico, la producción de información, modificaciones en las políticas locales y globales, y la interconexión, entre otros, que modifican la cultura, al trastocar las experiencias personales y las interpretaciones que los miembros de cada comunidad tienen estos cambios y la influencia que tienen en la concepción del tiempo, el espacio y las relaciones sociales. 
Sin embargo, ello ha repercutido en una exclusión de lo que Castells (2004) denominó cuarto mundo, es decir, áreas de ciudades, regiones o países que son irrelevantes para la mejora de la economía de aquellos cuya producción e ingresos va en aumento. De acuerdo con este autor, los elementos importantes ahora son la productividad y la competitividad, las cuales se miden a través de la innovación, la flexibilidad, el uso de las tecnologías de la información y comunicación y una capacidad para gestionar a través de redes. Las áreas que pertenecen al cuarto mundo, cada vez avanzan menos en el uso y producción de tecnología, de redes, y de conocimiento, y en consecuencia, dependen más de lo que hacen los países en desarrollo.

Ante esto, el tipo de ciudadano que la sociedad actual exige no es solo un trabajador genérico, como denomina Castells (2004) a aquel que almacena conocimientos y se queda obsoleto ante los cambios en la ciencia y tecnología, sino uno autoprogramable. Es decir, que puede ir evaluando los cambios que necesita hacer, las habilidades que debe desarrollar y los conocimientos que debe poseer para realizar nuevas tareas. Las diferencias entre ambos tipos de trabajadores, menciona el autor, es la educación, que se define como "...el proceso mediante el cual las personas, es decir los trabajadores, adquieren la capacidad de redefinir constantemente la cualificación necesaria para una tarea determinada y de acceder a las fuentes y métodos para adquirir dicha cualificación." (p.411).

Por otra parte, en el aspecto cultural, de acuerdo con Lechner (2002), las representaciones o imágenes de la convivencia social, al igual que la práctica de vivir con otros ha cambiado, lo cual tiene su repercusión en otro aspecto: el político. Lechner resalta que, con la cómoda accesibilidad a productos de cualquier país del mundo, así como la facilidad para comunicarse con personas de diferentes culturas, los límites que antes se establecían entre tiempo y espacio 
territorial han desaparecido. También, ello conlleva una mayor libertad para elegir, así como una visualización de más puntos de vista, lo que ha llevado a las personas a construir su propia identidad de manera más individualista que social, teniendo como consecuencia poca solidaridad y ausencia de identidad local.

Agregado a ello, los avances en la tecnología, la producción industrial y la libertad de actuar del hombre han llevado a éste a una nueva forma de interactuar con la naturaleza, que ha traído como consecuencia desastres ecológicos. Podría decirse que se ha invertido la relación hombre-naturaleza, ya que, de ser objeto de admiración y respeto, la naturaleza ha pasado a ser dominada por el hombre de acuerdo con sus intereses.

Según Fuentes (2002), el problema ecológico es uno de los principales asuntos que deben afrontarse a nivel mundial, puesto que el trato que se ha dado a la naturaleza y al ambiente está generando escasez de agua, emisiones masivas de dióxido de carbono, tala y quema de árboles, accidentes nucleares y extinción de especies. La forma de interactuar entre el hombre y la naturaleza puede armonizarse de nuevo con una formación distinta para las nuevas generaciones, la cual debe estar basada en el desarrollo de habilidades para pensar y actuar éticamente, lo cual constituye un reto para la educación.

Finalmente, para ubicar la situación contextual de hoy vale la pena mencionar otras características de la época, como lo son el desempleo, el subempleo, el aumento de personas excluidas, exiliadas, migrantes, la guerra económica entre países y bloques de poder, el agravamiento de la deuda externa, el comercio de armamento, el SIDA, la violencia y el contrabando de drogas, entre otros.

Si se espera que la situación mejore, desde el sector educativo se deben fortalecer acciones y procesos. Sin embargo, se debe partir de un conocimiento de 
la realidad que atraviesa la educación en Latinoamérica, la cual se caracteriza por desigualdades entre países, así como dentro de cada nación. Unas de las principales preocupaciones de las entidades internacionales que velan por la educación son el nivel de las tasas netas de matrícula, los porcentajes de niños con rezago académico y los diferentes niveles educativos que son alcanzados por la población.

De acuerdo con el informe Metas Educativas 2021 (OEI, 2010), para los años 2007-2008, en lberoamérica la tasa de cobertura para todos los países excepto uno, República Dominicana, era mayor al 90\%, lo cual demostró que casi la totalidad de niños en edad escolar se encontraban estudiando; sin embargo, estos datos no reflejaron la calidad de educación que estaban recibiendo. El mismo informe indicaba que entre los 9 y 11 años de edad, se observaban tasas de rezago escolar muy variadas entre los distintos países, que iban desde el $3 \%$ (México) hasta el 26\% (Guatemala) lo que representa un costo muy elevado para los países en los que se vive esta realidad.

No obstante, al avanzar al nivel de educación secundaria la tasa neta de matrícula varía mucho más de acuerdo con la región, y aunque el porcentaje general para Iberoamérica es de $85 \%$, existen países como Brasil, España y Chile, con porcentajes arriba de $94 \%$ y países, específicamente de la región centroamericana, como Honduras, El Salvador, Nicaragua y Guatemala, cuyos porcentajes oscilan entre el 55 y el $41 \%$ (OEI, 2010).

Asimismo, a nivel iberoamericano, en relación con el atraso escolar entre niños de 12 a 14 años, la tendencia es que sea mayor si el clima educativo del hogar es bajo y si el estudiante vive en una zona rural; y con respecto a género, se ha observado que es mayor el porcentaje de mujeres que de hombres que concluyen el nivel de secundaria. En relación con el nivel académico, también puede notarse 
que, dentro del sistema educativo de la Región Centroamericana, a mayor nivel educativo menor cantidad de personas que acceden a él.

Al respecto, si se analiza más allá del nivel de enseñanza básica o primaria, un nivel educativo más avanzado, como lo es la enseñanza secundaria alta o bachillerato, la tasa de matrícula en Iberoamérica cae hasta un 67\%, siendo Brasil, Cuba y Chile los países que poseen las mayores tasas de matrícula - 84\%, 83\% y $81 \%$ respectivamente- y nuevamente los países de la región centroamericana son los que poseen las tasas más bajas de matrícula neta: Guatemala, el Salvador y Nicaragua, con 33\%, 32\% y $15 \%$ respectivamente (OEI, 2010).

Al observar la situación sobre la cantidad de jóvenes de 25 a 29 años que finalizan 5 años de carrera universitaria, cabe destacar que, a nivel de lberoamérica, se cuenta con un 8.3\% de participación (OEI, 2010); y de ellos, por cada 27 jóvenes de ingresos económicos altos, sólo 1 de ingresos económicos bajos concluye los cinco años de universidad.

De acuerdo con los datos anteriores, la accesibilidad a la educación decae conforme el nivel educativo aumenta, y además, la probabilidad de que personas de escasos recursos terminen la universidad es muy baja en comparación con quienes tienen los recursos económicos necesarios. $Y$ si se considera que la educación es una forma de promover el desarrollo de una localidad, los sectores menos favorecidos seguirán manteniéndose así, a menos que aumenten las oportunidades de estudio y/o aumente la responsabilidad social de empresas y profesionales que han tenido la oportunidad de salir adelante.

Aunado a ello, existe otra problemática que aqueja la realidad educativa de Latinoamérica como lo es el deficiente aporte al desarrollo científico y tecnológico (Bello, 2001), así como a la investigación, tanto en esfuerzos como en resultados, 
que se ve reflejado en la escasa cantidad de investigaciones y en las reducidas disciplinas en las que se investiga. En este sentido, se observa que casi exclusivamente son las universidades las encargadas de realizar investigación, puesto que las empresas privadas están desligadas de estos procesos. Asimismo, las investigaciones, por lo general, están desvinculadas del sistema productivo y tienen poco impacto a nivel internacional.

Sin embargo, para que la educación cumpla con sus fines y avance en cobertura y calidad, es necesario contar con el recurso económico necesario para invertir en el funcionamiento del sistema. Knut (2000) ha señalado el impacto negativo que tiene un escaso presupuesto asignado al sector educativo, especialmente en los países centroamericanos, puesto que este factor incide en una insuficiente e inadecuada infraestructura para la educación y en una "pedagogía arcaica" que puede ser observada en muchos salones de clase, a través de una deficiente preparación de los profesores y escasez de materiales para la formación.

Ante esta realidad cabe preguntarse, ¿cuáles pueden ser las consecuencias de no promover la equidad en la educación, y con ello no intervenir en el desarrollo de la sociedad? ¿Quiénes son los responsables de ejecutar las acciones para que el ritmo de la educación mejore?

Las consecuencias de la falta de consciencia e intervención ante las desigualdades que se dan en Latinoamérica pueden aumentar la experiencia de una vida de pobreza, injusticia, violencia, exclusión e incluso de destrucción en la población. La participación para generar transformaciones sociales a través de la educación es fundamental, especialmente en Centroamérica, donde se necesitan ciudadanos que transformen sus regiones y que contribuyan a elevar la calidad de vida de sus habitantes. 
Para hacerlo, se necesita una base política, social y económica que garantice que las propuestas se llevarán a cabo. Al respecto, Lampert (2003) señala que en los países emergentes se hace más necesario que en los desarrollados la participación de los distintos sectores sociales, tanto gubernamentales como no gubernamentales, para que mejore la cobertura y calidad de la educación, y a su vez, repercuta en una educación que humanice a la población.

Es principio, los gobiernos deben tener una comunicación estrecha entre sí, para que sus esfuerzos puedan producir estrategias y recursos que ayuden a la región, no a naciones aisladas, puesto que en el contexto de la globalización se requieren metas comunes, aunque contextualizadas.

Por otro lado, se hace preciso asignar al rubro de la educación un gasto más congruente con las necesidades del país, ya que si no se invierte, la calidad de la educación no puede mejorar. Sin embargo, la gestión del dinero debe ser manejada de forma transparente, por personas comprometidas y profesionales que hagan un buen manejo del presupuesto.

Paralelo a los esfuerzos del Estado y de los ministerios de educación, los distintos sectores de la sociedad deben participar en la elaboración del proyecto educativo que desean. Es decir, no se deben dejar las decisiones educativas sólo en manos de los ministerios de educación, sino que las empresas privadas, las familias, los profesores e incluso organizaciones internacionales pueden participar en la planeación y ejecución de los cambios que se requieren, de acuerdo con las necesidades sentidas por las comunidades, y relacionadas con los acuerdos internacionales. Por mucho tiempo el poder ha estado en los altos mandos de los ministerios de educación, pero siendo la educación un derecho de la población, todos pueden intervenir en su mejora. 
Y si se cuenta con el apoyo de distintos sectores ¿cuál es el papel de la educación, en este momento? ¿Qué giros debe tomar para generar solidaridad, justicia y paz, y con ello conducir al progreso de nuestras naciones? ¿Qué tipo de hombre debe formar? ¿Cómo debe ser la relación entre educación, tecnología y conocimiento?

La educación debe orientar su paradigma, si bien es cierto, pero su fundamento debe alinearse con sus principales objetivos, como lo es formar un ciudadano más competente para cuidar, mejorar y transformar el mundo, para lo cual el Estado debe asegurar que la mayoría de personas pueda tener acceso a una educación de calidad donde el desarrollo de habilidades cognoscitivas, procedimentales y actitudinales lo impulsen a pensar y analizar con más criterio, creatividad y autonomía (Zubiría, 2005), a ser más participativo, democrático y justo, y a resguardar el bien común y la dignidad de las personas (Aguerrondo, 1999).

Para ello, se torna imperioso hacer cambios en la gestión, el currículum, la formación de profesores, la participación de la familia, la calidad de la educación y la evaluación de la misma, así como que se facilite el acceso a la tecnología y a la información, para que utilizados adecuadamente, se vuelvan promotores del crecimiento de los seres humanos, sin deteriorar las relaciones entre los mismos.

Esta demanda requiere que el paradigma educativo subyacente, apunte a una formación que favorezca la equidad social que constituye uno de los principales desafíos de la educación para este siglo (López, 2005). Además, de acuerdo con Taeli (2010), la educación debe basarse en el desarrollo de una consciencia de la complejidad de la realidad, de la interrelación entre sistemas y de que cada sujeto es parte de un contexto más amplio. Por lo que, se hará necesario formar no solo en competencias cognoscitivas y procedimentales, sino especialmente en las actitudinales. 
Asimismo, como la realidad es cambiante, la educación debe brindarse y autogestionarse durante toda la vida, por lo que, siguiendo la línea de Tedesco (2003), será necesario que las personas, a través del proceso educativo, aprendan a aprender (desafío cognoscitivo), y en segundo lugar, aprendan a vivir juntas (desafío social). Si se fortalecen estos aprendizajes, se puede incrementar la solidaridad y afirmación de los valores humanos, así como la capacidad de producir conocimiento y con ello, se contribuirá al progreso de la sociedad. Así, con estas herramientas, las nuevas generaciones podrán trabajar en planteamientos para lograr la equidad, la igualdad en el acceso a la educación, y la calidad de la misma.

Otro de los desafíos será impulsar el desarrollo de habilidades para que las personas sean capaces de enfrentar la incertidumbre. Para ello, será preciso que se conozca sobre el conocimiento humano, que se forme en aspectos de ética, que se desarrolle una consciencia individual, social y de especie, así como una consciencia planetaria. Será necesario estimular las habilidades cognoscitivas especialmente la comprensión, pues todo ello llevará al planteamiento de una educación que fomente la paz y la comprensión entre los seres humanos (Morín, 1999).

Para hacer de estas ideas una realidad, la gestión de la educación tiene que estar en manos de personas responsables, profesionales, con ética, que se comprometan con el desarrollo de su país y región. Asimismo, los cambios curriculares deberán basarse en acuerdos realizados entre países, pero con la capacidad de adaptarse a las necesidades de cada escuela.

En este proceso los profesores tendrán un papel importante, por lo que su formación deberá adaptarse al nuevo paradigma, y actualizarse constantemente, donde el uso de las tecnologías de la información y la comunicación tendrá un papel relevante. Los materiales y los medios para aprender podrán variar, pero los 
fines de la educación deberán mantenerse, por lo que la filosofía y las políticas educativas también deben redefinirse en función de ese ser humano que se desea formar, el cual, en el contexto de la sociedad actual debe aspirar a transformar el mundo para lograr la paz.

\section{Conclusiones}

Latinoamérica posee un rezago en los índices de progreso con respecto al nivel alcanzado por países desarrollados. Si se considera únicamente el factor educativo, hace falta ampliar la cobertura y la calidad, así como garantizar que haya más estudiantes que avancen a niveles educativos superiores, lo que puede ayudar a mejorar sus condiciones de vida y a participar de una manera más consciente en acciones que transformen su contexto.

La educación sigue siendo el sistema desde donde se puede impulsar la formación del ciudadano que se desea, con la capacidad para construir una sociedad equitativa y pacífica, donde sus habitantes puedan responder a las realidades complejas, trastocadas por los cambios que en diferentes dimensiones sociales devienen, cada vez, a un ritmo más acelerado, y que han trasformado la forma de vida y de relacionarse de las personas.

Para lograrlo, la comunidad y el sector empresarial deben intervenir brindando insumos al sector educativo para que la teoría y la práctica puedan servir al desarrollo. También deben permitirse espacios para la colaboración de las tres partes, donde cada uno pueda apoyar a otro y lograr procesos de formación, así como prácticas e intercambios que apunten a formar a los futuros ciudadanos de cada país, con aquellas competencias que se necesitan para mejorar la calidad de vida y para convivir en paz. Estas capacidades giran en torno a la tolerancia, el respeto a la dignidad de la persona, la convivencia pacífica, la participación 
ciudadana, el manejo de la incertidumbre, la producción de conocimiento y tecnología, y el aprender a aprender, que en su conjunto, contribuyen a que las personas vivan armoniosamente, en un ambiente sano y con oportunidades para vivir con un nivel adecuado de vida.

Con la priorización del gobierno sobre los fines de la educación, y su apoyo para alcanzarlos, la tarea de los gestores académicos y profesores se facilitará. Sin embargo, el compromiso y ética de ellos, son los que finalmente harán posible esta tarea. Por lo tanto, ser consciente de los fines de la educación es importante, pero para alcanzarlos, lo es más, que cada ciudadano se haga responsable de ellos, desde el espacio que le corresponde.

\section{Referencias}

Aguerrondo, I. (1999). El nuevo paradigma de la educación para el siglo. Argentina.

Bello, M. (2001). Reformas y Políticas Educativas en América Latina. Acción Pedagógica, 10 (1 y 2) 14-25.

Castells, M. (2004). La era de la información. Economía, sociedad y cultura. Fin de

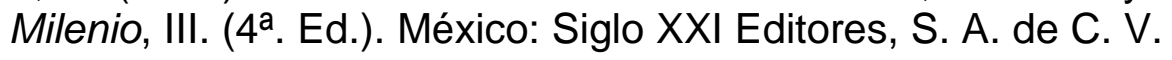

Fuentes, G. (2002). Ingeniería y Ética Profesional. Nueva ética para el siglo $X X I$. (1-16). Bilbao: RGM.

Knut, W. (2000). La educación en Centroamérica: reflexiones en torno a sus problemas y su potencial. Cuaderno de trabajo No.10. Oficina de Desarrollo Regional y Sostenible, América Latina y el Caribe (LAC) de la Agencia para el Desarrollo Internacional de los Estados Unidos y la Unión Europea.

Lampert, E. (2003). Educación: visión panorámica mundial y perspectivas para el siglo XXI. Perfiles educativos, 25(101), 7-22.

Lechner, N. (2002). Los desafíos políticos del cambio cultural. Nueva Sociedad 184. (p.46-65).

Recuperado 
de: http://www.insumisos.com/lecturasinsumisas/los\%20desafios\%20politico s\%20del\%20cambio\%20cultural\%20\%20\%20N\%20Lechner.pdf

López, N. (2005). La educación en América Latina, entre el cambio social y la inercia institucional. Revista Galega de Economía, 14(1-2), 1-20.

Morín, E. (1999). Los siete saberes necesarios para la educación del futuro. Francia: UNESCO.

Organización de Estados Iberoamericanos para la Educación, la Ciencia y la Cultura (OEI). (2010). Metas educativas 2021. La educación que queremos para la generación de los bicentenarios. España: Cudipal. Recuperado de: http://www.oei.es/metas2021.pdf

Taeli, F. (2010). El nuevo paradigma de la complejidad y la educación: una mirada histórica. Revista de la Universidad Bolivariana, 9(25), 183-198.

Tedesco, J. (2003). Los pilares de la educación del futuro. En: Debates de educación (2003: Barcelona) [ponencia en línea]. Fundación Jaume Bofill; UOC. Recuperada de: http://www.uoc.edu/dt/20367/index.html.

Zubiría, J. (2005). Los retos a la educación en el siglo XXI. España: Bonaventuriana. 